\title{
Theory of Enceladus and Dione
}

\author{
William H. Jefferys and Lynne M. Ries \\ University of Texas at Austin, Austin, Texas \\ (Received 24 June 1975)
}

\begin{abstract}
A new theory of the motion of Enceladus and Dione is under development. The theory is in literal form, with all constants and parameters appearing explicitly, and is being computed with the aid of the algebraic manipulation system TRIGMAN (Jefferys 1970, 1972). In this paper we treat in sections I-IV the development of the disturbing function; in sections V-VIII the elimination of the short-period terms; and in sections IX-XI the resonant Hamiltonian and the constants of integration. The theory has a number of novel features. One important feature is that the programs have been written in such a manner that, with only minor modification, they should be able to develop the theories of Mimas-Tethys and Titan-Hyperion as well.
\end{abstract}

\section{INTRODUCTION}

$I^{\mathrm{N}}$ $\mathrm{N}$ preparing for the exploration of the outer planets, it has become evident that our current knowledge about the orbits of their satellites is very poor indeed. The theories now in use date back to the early part of this century (Struve 1930, 1933; Woltjer 1928), and no longer provide accurate ephemerides. Errors in the predicted coordinates ranging into the tens of thousands of kilometers have been quoted (Mulholland 1972), principally due to the fact that, for the most part, these objects have been ignored for almost half a century. This has resulted in a dearth of recent, highly accurate observations. Since a primary purpose of the proposed missions has been to determine more about the positions and physical properties of the satellites of the outer planets, it is evident that an improvement in the orbits is highly desirable, even mandatory. Therefore, a two-pronged program has been undertaken simultaneously to obtain modern observations of high accuracy (Abbot, Mulholland, and Shelus 1975), and to develop new analytical theories of the motions of these objects.

Our aim in this and subsequent papers is to develop a new theory of the motion for each of three pairs of satellites in Saturn's system for which resonance effects are important: Mimas-Tethys, Enceladus-Dione, and Titan-Hyperion. Although the critical argument varies from case to case, it can be expected that similar problems will arise in the three cases, and hence the goal has been to treat all three cases with a common set of computer programs. We hope not only to be able to satisfy the demands of present and future Earth-based observations, but also those of observations from space vehicles. This implies an accuracy for the theory of the order of $1 \mathrm{~km}$ or so.

The main features of the theory are the following.

(1) It is being calculated with the aid of the algebraic manipulation system TRIGMAN (Jefferys 1970, 1972); TRIGMAN can handle up to ten trigonometric and 29 polynomial variables, more than adequate for this investigation;

(2) It is canonically invariant, as the Hori-Lie method (Hori 1966) has been used;
(3) The theory has been developed in terms of small quantities related to (but not identical with) the classical elements, i.e., semimajor axis, eccentricity, and sine inclination;

(4) Perturbations in any desired quantity can be computed very simply;

(5) Derivatives, as well as values, of any perturbed quantity with respect to the orbital elements and other parameters are provided for use in differential correction.

\section{VARIABLES}

We have used the following variables for the development of the Hamiltonian: the Delaunay angles $l$ (mean anomaly), $g$ (argument of pericenter), and $h$ (longitude of node), together with variables $A, E$, and $I$ as defined below. The variables for the inner and outer satellites have been distinguished by subscripts 1 and 2 , respectively.

If $a, e$, and $i$ are the usual elliptic elements (semimajor axis, eccentricity, and sine of the inclination), then the variables $A, E$, and $I$ are defined by the equations

$$
\begin{aligned}
a & =\bar{a}+f_{A} A, \\
e & =\bar{e}+f_{E} E, \\
i & =\bar{\imath}+f_{I} I,
\end{aligned}
$$

where $\bar{a}, \bar{e}$, and $\bar{\imath}$ are nominal numerical values chosen to agree approximately with the available mean orbits; and $f_{A}, f_{E}$, and $f_{I}$ are small numerical factors chosen so that the variables $A, E$, and $I$ will range over an interval of order unity as $a, e$, and $i$ range over all theories of practical interest. Of course, the quantities above are subscripted to correspond with the two satellites, but the subscripts are suppressed in this section.

As a special case, one evidently obtains the usual elements by setting $\bar{a}=\bar{e}=\bar{\imath}=0$ and $f_{A}=f_{E}=f_{I}=1$; the programs have been written so that this is, indeed, an option. However, there are a number of advantages to the above formulation when literal calculations on the computer are carried out.

Specifically, if any of the variables $a, e$, or $i$ is large, a power-series expansion in terms of the variable itself 
would converge slowly and contain many terms. This is particularly true of the semimajor axis, which is, of course, why direct literal expansions in this variable have been attempted so seldom. On the other hand, the use of a nominal value, say $\bar{a}$, and a variable $A$ allows the expansion in terms of $A$ to be carried only to very low orders. This can produce a more compact theory, as was shown by Lieske (1974) in his rediscussion of Sampson's theory of the Galilean satellites.

Another advantage lies in the area of truncation. In an expansion in the above variables, each term will, in addition to having an associated monomial (say, $A^{j} E^{k} I^{l}$ ), be factored by an associated product of small factors (e.g., $f_{A}^{j} f_{E}^{k} f_{I}^{l}$ ) and a floating-point coefficient results. The TRIGMAN system can truncate a term if either one of two conditions is satisfied: First, if a weighted sum of the exponents of a term is greater than a present quantity, that is,

$$
w_{A}^{j}+w_{E}^{k}+w_{I}^{\imath}>n,
$$

where $w_{A}, w_{E}$, and $w_{I}$ are integers; and second, if the coefficient of a term is less than a preset tolerance. By judicious choice of the tolerance, of the factors $f_{A}, f_{E}$, and $f_{I}$, and of the weights $w_{A}, w_{E}$, and $w_{I}$, it has been found possible to maintain much closer control over which terms are retained in a series and which ones are dropped. This, in turn, leads to a more compact theory. The use of scaling factors has been suggested by Broucke (1971) in a somewhat different context.

As noted above, our guiding principle for choosing $f_{A}, f_{E}, f_{I}$, and the tolerance has been the observation that for an appropriate choice of each factor the associated variable will range over an interval of order unity, while encompassing all theories of practical interest. An appropriate choice is, in fact, equal to the expected range of a particular variable, so that, e.g., in the expression $a=\bar{a}+f_{A} A$, if $f_{A}$ is chosen somewhat larger than the uncertainty in $A$, then varying $A$ in a range of order unity will certainly include any theory of interest. We then set the truncation parameter so that terms an order of magnitude smaller than any expected to be of importance are retained.

\section{THE DIRECT PART OF THE DISTURBING FUNCTION}

The principal problem in the development of the disturbing function is, of course, the development of the direct part $1 / \Delta$, where

$$
\Delta^{2}=r_{1}^{2}+r_{2}^{2}-2 r_{1} r_{2} \cos S,
$$

$r_{1}$ and $r_{2}$ are the distances of each satellite from the primary, and $S$ is the angle between the two radius vectors. This must be developed in terms of the aforementioned variables $\left(l_{j}, g_{j}, h_{j}, A_{j}, E_{j}, I_{j}\right)$ describing the two orbits.

We have developed a computer program to accomplish this by a relatively straightforward method. It accepts as input nominal (barred) values of the follow- ing quantities: the mass of Saturn and of each satellite, the mean motion in mean longitude of each satellite, the eccentricities and sine inclinations $e$ and $i$, and the dynamical form factors $J_{2}$ and $J_{4}$ of Saturn. From these it computes values for $\bar{a}$ by correcting the mean motion of the mean longitude for the secular effects of the oblateness and then applying Kepler's third law. Then, with the factors $f_{A}, f_{E}$, and $f_{I}$ for each satellite, explicit expressions for $a_{j}, e_{j}$, and $i_{j}$ are calculated for each satellite using Eq. 1. From this point on, all expansions are in terms of the variables $A_{j}, E_{j}$, and $I_{j}$.

The first step of the development is to derive expressions for the standard functions in elliptic motion such as the radius $r$, the equation of the center $f-l$ (where $f$ is the true anomaly), etc., in terms of the above variables. Following Barton (1966), this has been done directly from their definitions. We first solve Kepler's equation by using

$$
\varphi^{(n+1)}=e \sin \left(l+\varphi^{(n)}\right)
$$

where $\varphi=u-l$ and $u$ is the eccentric anomaly, expanding the sine in Taylor's series, and iterating until convergence is obtained. This is easily accomplished using the TRIGMAN function SUBST. We then compute

$$
r / a=1-e \cos (l+\varphi),
$$

by series substitution, and

$$
a / r=(r / a)^{-1}
$$

by expansion in geometric series. Also,

$$
f-l=\int\left[\left(1-e^{2}\right)^{\frac{1}{2}}(a / r)^{2}-1\right] d l
$$

is calculated using the series integration routine. We also calculate for later use other similar expressions, such as the expression for $r$ in terms of the eccentric anomaly and of $1 / r$ in terms of the true anomaly (both of which are closed-form expressions in the angular argument).

Having taken care of the required formulae of elliptic motion, we then compute an expression for $\cos S$ (Fig. 1). We have

$$
\begin{aligned}
& x=\cos (v+h) \cos \beta, \\
& y=\sin (v+h) \cos \beta, \\
& z=\sin \beta
\end{aligned}
$$

for each satellite. In addition, we have from spherical trigonometry,

$$
\begin{aligned}
\cos (f+g) & =\cos \beta \cos v, \\
\left(1-i^{2}\right)^{\frac{1}{2}} \sin (f+g) & =\cos \beta \sin v, \\
i \sin (f+g) & =\sin \beta
\end{aligned}
$$

remembering that $i$ is the sine of the inclination. Com- 


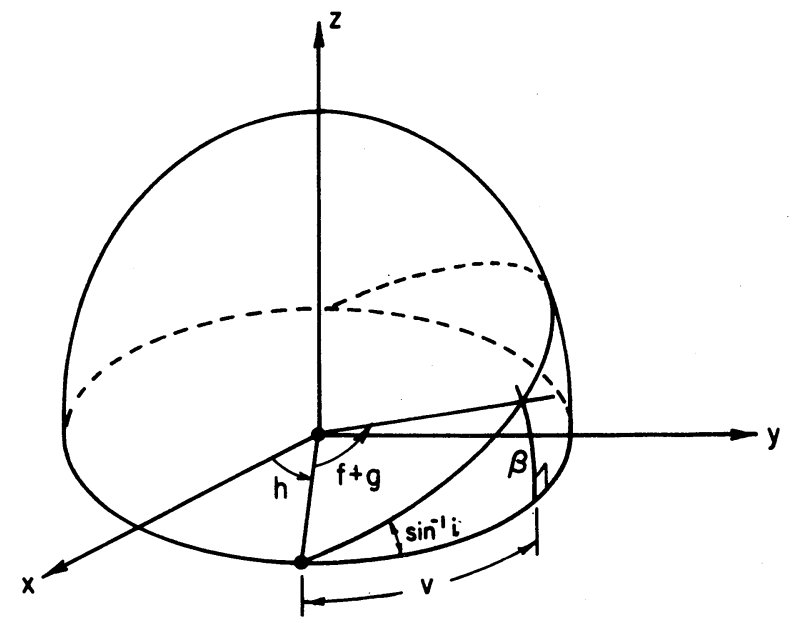

FIG. 1. Geometry for the calculation of rectangular coordinates of a satellite.

bining Eqs. 8 and 9 we obtain

$$
\begin{aligned}
& x=\cos (f+g) \cos h-\left(1-i^{2}\right)^{\frac{1}{2}} \sin (f+g) \sin h, \\
& y=\cos (f+g) \sin h+\left(1-i^{2}\right)^{\frac{1}{2}} \sin (f+g) \cos h, \\
& z=i \sin (f+g)
\end{aligned}
$$

for each satellite, as series in $f, g, h$, and $I$. Finally, we have

$$
\cos S=x_{1} x_{2}+y_{1} y_{2}+z_{1} z_{2},
$$

which is a completely general expression allowing the calculation of $\cos S$ in an arbitrary coordinate system (here, the equatorial plane of Saturn). For convenience, at this point we express $r_{1} r_{2}{ }^{-1} \cos S$ in terms of the eccentric anomaly of the inner body and the true anomaly of the outer body, since this yields an expression in closed form.

The next step in the development of the series is to determine an initial approximation involving Laplace coefficients. Instead of computing Laplace coefficients in the usual fashion, however, we have chosen to work directly from the definition of the disturbing function. Setting

$$
\left(\Delta_{0} / \bar{a}_{2}\right)^{2}=1+\bar{\alpha}^{2}-2 \bar{\alpha} \cos \Sigma,
$$

where

$$
\bar{\alpha}=\bar{a}_{1} / \bar{a}_{2}
$$

and $\Sigma$ is an angle (equivalent to $S$ eventually), we compute

$$
\Phi_{0}=\bar{a}_{2} / \Delta_{0}
$$

by a method advocated by Broucke (1971). Specifically, we assume a first approximation for $\Phi_{0}\left[\right.$ here, $\left.\left(1+\bar{\alpha}^{2}\right)^{-\frac{1}{2}}\right]$, and then iterate the equation

$$
\Phi_{0}^{(n+1)}=\Phi_{0}^{(n)}\left\{1+\frac{1}{2}\left[1-\left(\Delta_{0} / \bar{a}_{2}\right)^{2}\left(\Phi_{0}{ }^{(n)}\right)^{2}\right]\right\}
$$

until convergence is obtained. This is an adaptation of the Newton-Raphson method which is suitable for series expansion.
Next, defining

$$
\left(\Delta / r_{2}\right)^{2}=1+\left(r_{1} / r_{2}\right)^{2}-2\left(r_{1} / r_{2}\right) \cos S,
$$

which is readily expressed as a series in $u_{1}$ and $f_{2}$ with what we already have, a similar iteration with $\Phi_{0}$ as the initial approximation, and $\Sigma=u_{1}+g_{1}+h_{1}-f_{2}-g_{2}-h_{2}$ yields

$$
\Phi=r_{2} / \Delta .
$$

Multiplication by $1 / r_{2}$ followed by two substitutions using the already computed series $u_{1}-l_{1}$ and $f_{2}-l_{2}$ finally yields the desired result, expressed in terms of $\left(l_{j}, g_{j}, h_{j}, A_{j}, E_{j}\right.$, and $\left.I_{j}\right)$.

\section{THE REST OF THE DISTURBING FUNCTION}

The other terms in the disturbing functions are very straightforward, as most of the work has already been done. The indirect part is

$$
r_{1} r_{2}{ }^{-2} \cos S
$$

and can be calculated in terms of $u_{1}$ and $f_{2}$ with series already available; simple substitution then produces a series in terms of the mean anomalies. Likewise, the principal term in the oblateness has (apart from numerical factors) the form

$$
r_{j}^{-3} P_{2}\left(\sin \beta_{j}\right)
$$

where $\beta_{j}$ is the latitude and $P_{2}$ the Legendre polynomial of order 2. From Eqs. 9 we have that

$$
\sin \beta_{j}=i_{j} \sin \left(f_{j}+g_{j}\right)
$$

so that this, too, is easily computed from what already is available in like fashion. The complete form for the oblateness terms is given later in this paper.

\section{ADVANTAGES OF THE METHOD}

The development of the disturbing function in the variables $A, E$, and $I$ instead of the classical variables has one great advantage, namely, that fewer terms may be required to represent the final result adequately. The reason is that, even if the eccentricities and inclinations are large, if their variations are small, only a few powers of $E$ and $I$ need be computed, instead of the high powers of $e$ and $i$ that would be required.

An important feature of these variables is that the expressions for the partial derivatives are particularly simple: In fact, we have

$$
\begin{gathered}
\partial / \partial a=f_{A}^{-1} \partial / \partial A, \\
\partial / \partial e=f_{E}^{-1} \partial / \partial E, \\
\partial / \partial i=f_{I}^{-1} \partial / \partial I,
\end{gathered}
$$

so that the equations of motion bear a simple relationship to the classical ones. Similarly, if canonical variables are required (for example, if a Lie series method 
is to be employed) then we can write (say)

$$
\begin{aligned}
& L=\bar{L}+\mathcal{L}, \\
& G=\bar{G}+\mathcal{G}, \\
& H=\bar{H}+\mathfrak{H},
\end{aligned}
$$

and the set $(\mathcal{L}, \mathcal{G}, \mathfrak{F}, l, g, h)$ is again canonical. Notice that the variables $\mathscr{L}, \mathcal{G}$, and $\mathfrak{H}$ bear a simple relationship to $A, E$, and $I$. We have, for example,

$$
\begin{aligned}
& A=\left(\frac{2 \bar{L}}{\mu} \mathfrak{L}+\frac{\mathfrak{L}^{2}}{\mu}\right) f_{A}^{-1}, \\
& \mathscr{L}=\frac{1}{2}\left(\frac{\mu}{\bar{a}}\right)^{\frac{1}{2}} f_{A} A+\cdots,
\end{aligned}
$$

and similarly for the other variables. These expansions can easily be carried out to any order of accuracy on the computer.

\section{EQUATIONS OF MOTION}

In Jacobi coordinates (Fig. 2), the equations of motion for the three-body problem can be written (Jefferys and Moser 1966)

where

$$
\tilde{\nu}_{j} \mathbf{r}_{j}=\partial \tilde{U} / \partial \mathbf{r}_{j}, \quad j=1,2,
$$

$$
\begin{aligned}
& \tilde{\nu}_{1}=m_{0} m_{1} /\left(m_{0}+m_{1}\right), \\
& \tilde{\nu}_{2}=m_{2}\left(m_{0}+m_{1}\right) /\left(m_{0}+m_{1}+m_{2}\right),
\end{aligned}
$$

and

$$
\tilde{U}=k^{2}\left(\frac{m_{0} m_{1}}{r_{01}}+\frac{m_{0} m_{2}}{r_{02}}+\frac{m_{1} m_{2}}{r_{12}}\right) .
$$

The subscripts 0,1 , and 2 refer in this case to Saturn, the inner satellite and the outer satellite, respectively. The Gaussian constant of gravity is $k$, the masses are $m_{j}$, and the Jacobi relative coordinates are $\mathbf{r}_{j}$.

From the diagram,

$$
r_{02}^{2}=\left(\frac{m_{1}}{m_{0}+m_{1}}\right)^{2} r_{1}^{2}+r_{2}^{2}+2\left(\frac{m_{1}}{m_{0}+m_{1}}\right) r_{1} r_{2} \cos S
$$

so that, by the usual expansion in Legendre polynomials,

$$
\begin{aligned}
\frac{1}{r_{02}} & =\frac{1}{r_{2}}\left[1-\left(\frac{m_{1}}{m_{0}+m_{1}}\right) \frac{r_{1}}{r_{2}} \cos S\right]+O\left(m_{1}^{2}\right), \\
& =\frac{1}{r_{2}}-\frac{m_{1}}{m_{0}} \frac{r_{1}}{r_{2}^{2}} \cos S+O\left(m_{1}^{2}\right),
\end{aligned}
$$

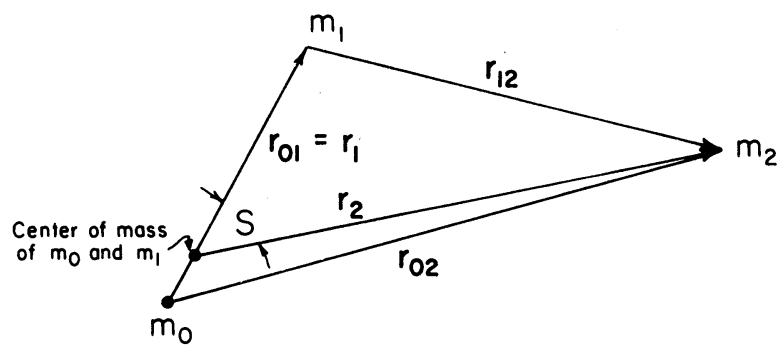

FIG. 2. Jacobi coordinates for the three-body problem.

and the expression for $\tilde{U}$ becomes

$$
\begin{aligned}
\tilde{U} & =k^{2}\left\{\frac{m_{0} m_{1}}{r_{01}}+\frac{m_{0} m_{2}}{r_{2}}+m_{2}\left[\frac{m_{1}}{r_{12}}+m_{0}\left(\frac{1}{r_{02}}-\frac{1}{r_{2}}\right)\right]\right\} \\
& =k^{2}\left\{\frac{m_{0} m_{1}}{r_{1}}+\frac{m_{0} m_{2}}{r_{2}}+m_{1} m_{2}\left(\frac{1}{\Delta}-\frac{r_{1}}{r_{2}^{2}} \cos S\right)\right\},
\end{aligned}
$$

where terms in the cube of the masses $m_{1}, m_{2}$ have been neglected, and we have written $r_{01}=r_{1}$ and $r_{12}=\Delta$.

The problem has now been reduced to the form of two perturbed, two-body problems, where the mutual perturbation is given by the disturbing function

$$
\tilde{U}_{1}=k^{2} m_{1} m_{2}\left(\frac{1}{\Delta}-\frac{r_{1}}{r_{2}^{2}} \cos S\right)
$$

In addition, we have the contribution of the oblateness to consider, which is given by

$$
\tilde{U}_{2}=-k^{2} m_{0} \sum_{j=1}^{2} \frac{m_{j}}{r_{j}} \sum_{n=2}^{\infty} \frac{J_{n} R^{n}}{r_{j}^{n}} P_{n}\left(\sin \beta_{j}\right),
$$

where $R$ is the equatorial radius of Saturn. Going over to Delaunay variables we have for the Hamiltonian

$$
\widetilde{F}=\frac{\mu_{1}^{2} \tilde{\nu}_{1}^{3}}{2 \tilde{L}_{1}^{2}}+\frac{\mu_{2}^{2} \tilde{\nu}_{2}^{3}}{2 \tilde{L}_{2}^{2}}+\tilde{U}_{1}+\tilde{U}_{2}
$$

and have defined

$$
\begin{aligned}
& \mu_{j}=k^{2} m_{0} m_{j} / \tilde{\nu}_{j}, \\
& \tilde{L}_{j}=\tilde{\nu}_{j}\left(\mu_{j} a_{j}\right)^{\frac{1}{2}}, \\
& \widetilde{G}_{j}=\tilde{L}_{j}\left(1-e_{j}\right)^{\frac{1}{2}} \\
& \tilde{H}_{j}=\widetilde{G}_{j}\left(1-i_{j}{ }^{2}\right)^{\frac{1}{2}}
\end{aligned}
$$

The angular variables conjugate to $\left(\tilde{L}_{j}, \widetilde{G}_{j}, \tilde{H}_{j}\right)$ are $\left(l_{j}, g_{j}, h_{j}\right)$, respectively.

Because of the smallness of the masses, the variables $\tilde{L}_{j}, \widetilde{G}_{j}, \widetilde{H}_{j}$, and the Hamiltonian $\widetilde{F}_{j}$ are inconveniently small. Hence, we have divided through by a suitably chosen small constant $\epsilon$ so that everything becomes of 
order unity. The resulting system of equations is

$$
F=\frac{\mu_{1}{ }^{2} \nu_{1}{ }^{3}}{2 L_{1}{ }^{2}}+\frac{\mu_{2}{ }^{2} \nu_{2}{ }^{3}}{2 L_{2}{ }^{2}}+U_{1}+U_{2},
$$

where

$$
\begin{aligned}
F & =\widetilde{F} / \epsilon, \\
\nu_{j} & =\tilde{\nu}_{j} / \epsilon, \\
L_{j} & =\tilde{L}_{j} / \epsilon, \\
G_{j} & =\tilde{G}_{j} / \epsilon, \\
H_{j} & =\tilde{H}_{j} / \epsilon, \\
U_{j} & =\tilde{U}_{j} / \epsilon .
\end{aligned}
$$

This gives a canonical transformation, provided that the angular variables remain $\left(l_{j}, g_{j}, h_{j}\right)$.

\section{COMPUTATION OF THE HAMILTONIAN}

For simplicity we have chosen a system of units based on Saturn's radius and mass, that is,

with

$$
\begin{gathered}
m_{0}=1, \\
R=1,
\end{gathered}
$$

$$
k=36.20776772 \text {, }
$$

so that the unit of time is the ephemeris day. The value of $k$ chosen is consistent with proposed IAU values of $299792458 \mathrm{~m} / \mathrm{sec}$ for the speed of light, 149597872 $\mathrm{km}$ for the astronomical unit, $60000 \mathrm{~km}$ for the radius of Saturn, and 3498.5 for the reciprocal mass of Saturn.

The Hamiltonian is required as a function of the masses and of $J_{2}$ and $J_{4}$, the oblateness parameters, so that the solution can be used to improve these values. Analogously to what has already been done with the elements, we set

and

$$
m_{j}=\bar{m}_{j}+f_{M_{j}} M_{j}
$$

$$
J_{j}=\bar{J}_{j}+f_{\Delta J_{j}} \Delta J_{j},
$$

so that our variables become $M_{j}$ and $\Delta J_{j}$. As before, the barred values are nominal values known in advance, and the factors $f_{M_{j}}$ and $f_{\Delta J_{j}}$ are chosen to make the variables $M_{j}$ and $\Delta J_{j}$ range over an interval of order unity.

The parameters $\nu_{j}$ and $\mu_{j}$ are determined as functions of the $M_{j}$, and these in turn are used to compute series for the canonical variables $L_{j}, G_{j}$, and $H_{j}$ in terms of $M_{j}, A_{j}, E_{j}$, and $I_{j}$. From these we then determine the Keplerian part of the Hamiltonian, $\Sigma_{\mu_{j}}{ }^{2} \nu_{j}{ }^{3} L_{j}{ }^{-2}$, also as series in these variables. To this we add the series for $U_{1}$ and $U_{2}$, obtained from the results of the program described earlier. The resulting Hamiltonian is used as the basis of all subsequent calculations.

\section{PARTIAL DERIVATIVES AND POISSON BRACKETS}

The Poisson bracket is a coordinate-independent quantity, and the Hori-Lie method is based on this fact (Hori 1966). However, the series used in calculations are always presented in some particular coordinate system. Hence, it is necessary to provide a method to compute the Poisson bracket of any two desired functions, in terms of the adopted coordinate system (which need not be a canonical coordinate system).

Our coordinate system is the set of variables $\left(A_{j}, E_{j}, I_{j}, l_{j}, g_{j}, h_{j}\right)$, and by definition, the Poisson bracket is

$$
\begin{array}{r}
\{p, q\}=\sum_{j=1}^{2}\left(\frac{\partial p}{\partial L_{j}} \frac{\partial q}{\partial l_{j}}-\frac{\partial p}{\partial l_{j}} \frac{\partial q}{\partial L_{j}}+\frac{\partial p}{\partial G_{j}} \frac{\partial q}{\partial g_{j}}-\frac{\partial p}{\partial g_{j}} \frac{\partial q}{\partial G_{j}}\right. \\
\left.+\frac{\partial p}{\partial H_{j}} \frac{\partial q}{\partial h_{j}}-\frac{\partial p}{\partial h_{j}} \frac{\partial q}{\partial H_{j}}\right)
\end{array}
$$

In order to compute this, the operators $\partial / \partial L_{j}, \partial / \partial G_{j}$, and $\partial / \partial H_{j}$ must be made available (in addition to the trivial derivatives with respect to the angles). One has

$$
\begin{aligned}
\frac{\partial}{\partial L_{j}} & =\frac{\partial A_{j}}{\partial L_{j}} \frac{\partial}{\partial A_{j}}+\frac{\partial E_{j}}{\partial L_{j}} \frac{\partial}{\partial E_{j}}, \\
\frac{\partial}{\partial G_{j}} & =\frac{\partial E_{j}}{\partial G_{j}} \frac{\partial}{\partial E_{j}}+\frac{\partial I_{j}}{\partial G_{j}} \frac{\partial}{\partial I_{j}}, \\
\frac{\partial}{\partial H_{j}} & =\frac{\partial I_{j}}{\partial H_{j}} \frac{\partial}{\partial I_{j}},
\end{aligned}
$$

where

$$
\begin{aligned}
& \frac{\partial A_{j}}{\partial L_{j}}=\frac{2 L_{j}}{\mu_{j} \nu_{j}^{2} f_{A_{j}}}, \\
& \frac{\partial E_{j}}{\partial G_{j}}=-\frac{\left(1-e_{j}^{2}\right)^{\frac{1}{2}}}{L_{j} e_{j} f_{E_{j}}}, \\
& \frac{\partial E_{j}}{\partial L_{j}}=-\left(1-e_{j}{ }^{2}\right)^{\frac{1}{2}} \frac{\partial E_{j}}{\partial G_{j}}, \\
& \frac{\partial I_{j}}{\partial H_{j}}=-\frac{\left(1-i_{j}{ }^{2}\right)^{\frac{1}{2}}}{G_{j} i_{j} f_{I_{j}}}, \\
& \frac{\partial I_{j}}{\partial G_{j}}=-\left(1-i_{j}{ }^{2}\right)^{\frac{1}{2}} \frac{\partial I_{j}}{\partial H_{j}} .
\end{aligned}
$$

These partial derivatives are computed as series in all variables at the outset, and subroutines have been provided to determine the partial derivative of any function with respect to any variable, using Eqs. 39, and the Poisson bracket of any two functions, from Eq. 38.

\section{NONRESONANT PERTURBATIONS}

According to Hori (1966), a generating function $W$, which is a function of new (primed) variables, pro- 
duces a canonical transformation of a function of new variables to the same function, expressed in old variables, by means of the formula

$$
p(\mathbf{x}, \mathbf{y})=p\left(\mathbf{x}^{\prime}, \mathbf{y}^{\prime}\right)+\{p, W\}+\frac{1}{2 !}\{\{p, W\}, W\}+\cdots
$$

where $(\mathbf{x}, \mathbf{y})$ and $\left(\mathbf{x}^{\prime}, \mathbf{y}^{\prime}\right)$ are the old and new canonical variables.

In particular, the Hamiltonian $F$ can be transformed:

$$
\begin{aligned}
F(\mathbf{x}, \mathbf{y}) & =F\left(\mathbf{x}^{\prime}, \mathbf{y}^{\prime}\right)+\{F, W\}+\cdots \\
& =F^{\prime}\left(\mathbf{x}^{\prime}, \mathbf{y}^{\prime}\right),
\end{aligned}
$$

where $F^{\prime}$, the new Hamiltonian, is unchanged in value because the transformation is canonical. Letting $F=F_{0}+F_{1}+\cdots, W=W_{1}+\cdots, F^{\prime}=F_{0}{ }^{\prime}+F_{1}{ }^{\prime}+\cdots$, we obtain to first order

$$
\begin{aligned}
F_{0}\left(\mathbf{x}^{\prime}, \mathbf{y}^{\prime}\right) & =F_{0}{ }^{\prime}\left(\mathbf{x}^{\prime}, \mathbf{y}^{\prime}\right), \\
F_{1}\left(\mathbf{x}^{\prime}, \mathbf{y}^{\prime}\right)+\left\{F_{0}, W_{1}\right\} & =F_{1}{ }^{\prime}\left(\mathbf{x}^{\prime}, \mathbf{y}^{\prime}\right)
\end{aligned}
$$

the second equation of which is a partial differential equation to be solved for $W_{1}$ and $F_{1}{ }^{\prime}$.

The splitting of $F$ into parts of various order is more or less arbitrary and has to be performed with the individual problem in mind. In our case, there are known to be terms in a critical argument $\theta$, where

$$
\theta=l_{1}+2 g_{1}+2 h_{1}-2 l_{2}-2 g_{2}-2 h_{2} \text {. }
$$

These terms must be isolated from the rest, since $\theta$ is known to librate about 0 . If we write

$$
F=P_{0}+P_{1}(\theta)+P_{2}\left(l_{j}, g_{j}, h_{j}\right),
$$

where $P_{0}$ is independent of the angles, $P_{1}$ contains all the critical terms in $\theta$, and $P_{2}$ is purely periodic, then we have chosen to set

$$
\begin{aligned}
& F_{0}=P_{0}+P_{1}(0), \\
& F_{1}=F-F_{0},
\end{aligned}
$$

in Eqs. 43 , since the libration is about $\theta=0$. This rather unusual decomposition guarantees that the nominal mean motions will be approximately correct to first order, and in particular that $\dot{\theta} \approx 0$ (as has been verified by our calculations). Note that $F_{0}$ will contain all terms that (to first order) contribute to the secular motion of the angles, including those arising from $U_{1}$ and $U_{2}$.

With this decomposition, the partial differential equation of Eq. 43 becomes

$$
F_{1}\left(\mathbf{x}^{\prime}, \mathbf{y}^{\prime}\right)-\sum_{j} n_{j} \frac{\partial W_{1}}{\partial y_{j}^{\prime}}=F_{1}^{\prime}\left(\mathbf{x}^{\prime}, \mathbf{y}^{\prime}\right)
$$

where

$$
n_{j}=-\frac{\partial F_{0}}{\partial x_{j}^{\prime}}
$$

are the mean motions of the angular variables. Equa- tion 47 can be solved in the special case that the function $F_{1}-F_{1}{ }^{\prime}$ is a purely periodic function of the angular variables. In order to accomplish this, $F_{1}{ }^{\prime}$ is usually chosen to contain all terms of $F_{1}$ independent of $\mathbf{y}^{\prime}$, plus any deeply resonant terms (i.e., terms in $\theta$ ). Then, $\left(F_{1}-F_{1}{ }^{\prime}\right)$ is of the form

$$
\begin{array}{r}
F_{1}-F_{1}{ }^{\prime}=\sum^{\prime}\left[C_{j_{1} \cdots j_{k}} \cos \left(j_{1} y_{1}+\cdots+j_{k} y_{k}\right)\right. \\
\left.+S_{j_{1} \cdots j_{k}} \sin \left(j_{1} y_{1}+\cdots+j_{k} y_{k}\right)\right],
\end{array}
$$

where the prime on the summation sign indicates the omission of secular and critical terms. The solution for $W_{1}$ is

$$
\begin{aligned}
W_{1}= & \sum^{\prime}\left[\frac{C_{j_{1} \cdots j_{k}}}{j_{1} n_{1}+\cdots+j_{k} n_{k}} \sin \left(j_{1} y_{1}+\cdots+j_{k} y_{k}\right)\right. \\
& \left.-\frac{S_{j_{1} \cdots j_{k}}}{j_{1} n_{1}+\cdots+j_{k} n_{k}} \cos \left(j_{1} y_{1}+\cdots+j_{k} y_{k}\right)\right] .
\end{aligned}
$$

Unfortunately, at this point a difficulty arises: TRIGMAN cannot handle a series of the form of Eq. 50 , because TRIGMAN is a Poisson series processor, and literal denominators of the form shown cannot be expressed. If the mean motions $n_{1}, \ldots, n_{k}$ were numbers, there would be no difficulty, and in fact, there exists a TRIGMAN library function PDES $\varnothing \mathrm{LV}$, which automatically solves an equation of the form of Eq. 47 for $W_{1}$, deciding on the basis of the size of $\left(j_{1} n_{1}+\cdots+j_{k} n_{k}\right)$ which terms belong in the new Hamiltonian $F_{1}{ }^{\prime}$, provided that $n_{1}, \ldots, n_{k}$ are real numbers.

This difficulty is circumvented in the following manner: The mean motions $n_{1}, \ldots, n_{k}$ are computed from $F_{0}$ using Eq. 48 ; the resulting values are series in $A_{j}, E_{j}, I_{j}, M_{j}, \Delta J_{j}$, with constant numerical terms $\bar{n}_{1}, \ldots, \bar{n}_{k}$ which are, in fact, the nominal values of the respective mean motions. Setting

and

$$
W_{1}=W_{1}^{(0)}+W_{1}^{(1)}+W_{1}^{(2)}+\cdots
$$

$$
n_{j}=\bar{n}_{j}+N_{j}
$$

we obtain from Eq. 47

$$
\sum_{j} \bar{n}_{j} \sum_{k} \frac{\partial W_{1}^{(k)}}{\partial y_{j}^{\prime}}=\left(F_{1}-F_{1}{ }^{\prime}\right)-\sum_{j} N_{j} \sum_{k} \frac{\partial W_{1}^{(k)}}{\partial y_{j}^{\prime}} .
$$

Assuming that the nominal values of the mean motions are approximately correct, and that the terms $N_{j}$ are small, we can split this equation into a hierarchy of equations :

$$
\begin{aligned}
& \sum_{j} \bar{n}_{j} \frac{\partial W_{1}^{(0)}}{\partial y_{j}^{\prime}}=\left(F_{1}-F_{1}{ }^{\prime}\right), \\
& \sum_{j} \bar{n}_{j} \frac{\partial W_{1}^{(k)}}{\partial y_{j}^{\prime}}=-\sum_{j} N_{j} \frac{\partial W_{1}^{(k-1)}}{\partial y_{j}^{\prime}}, \quad k=1,2, \ldots
\end{aligned}
$$


Each of these equations is now in a form acceptable to PDES $\emptyset \mathrm{LV}$. Therefore, Eq. 53 is used initially to solve for $W_{1}{ }^{(0)}$ and $F_{1}{ }^{\prime}$, and then Eq. 54 is used to solve successively for $W_{1}^{(1)}, W_{1}^{(2)}, \ldots$, until convergence is obtained (e.g., a $W_{1}{ }^{(k)}$ is computed which truncates to zero). This procedure works very well, and the new Hamiltonian selected by PDES $\emptyset \mathrm{LV}$ contains precisely the terms one would expect, namely, the secular terms and the terms in the critical argument $\theta$.

It is, by the way, absolutely necessary to take into account the terms arising from $W_{1}{ }^{(1)}, W_{1}{ }^{(2)}, \ldots$, in the determining function. Without them, it is easily shown that the perturbations which are calculated are incorrect. The reason for this is that important perturbations (particularly in the longitude) arise from the partial derivatives of the divisors $j_{1} n_{1}+\cdots+j_{k} n_{k}$ when the Poisson bracket $\left\{p, W_{1}\right\}$ is computed (see below). These are the same terms which are introduced in the classical Lagrangian planetary equations through the equation $d n / d t=-(3 n / 2 a) d a / d t$.

The new Hamiltonian $F_{0}{ }^{\prime}+F_{1}{ }^{\prime}$ now becomes the input to the next step in the theory, the solution of the resonant part of the Hamiltonian. The determining function $W_{1}$ is the source of all short-period and shallowly resonant perturbations, according to the prescription of the Hori-Lie method. Indeed, if $p\left(\mathbf{x}^{\prime}, \mathbf{y}^{\prime}\right)$ is any function of elliptic motion, the osculating value of $p$ is determined to first order by Eq. 55 :

$$
p(\mathbf{x}, \mathbf{y})=p\left(\mathbf{x}^{\prime}, \mathbf{y}^{\prime}\right)+\left\{p, W_{1}\right\} .
$$

Since a routine for computing Poisson brackets in our adopted coordinate system is available, it is only required that the desired functions $p$ be chosen and computed in these coordinates, and then for the Poisson bracket routine to be called. In principle, any desired perturbations can be computed; initially, we have determined them for the longitude $\psi=f+g+h$, the distance from the primary $r$, and the latitude $\beta$ of each satellite, as these quantities are readily compared with observations.

\section{THE REDUCED HAMILTONIAN}

The calculations described above have resulted in a new Hamiltonian of the form

$$
F^{\prime}\left(L_{j}{ }^{\prime}, G_{j}{ }^{\prime}, H_{j}{ }^{\prime}, \theta^{\prime}\right)
$$

where $\theta^{\prime}=l_{1}{ }^{\prime}+2 g_{1}{ }^{\prime}+2 h_{1}{ }^{\prime}-2 l_{2}{ }^{\prime}-2 g_{2}{ }^{\prime}-2 h_{2}{ }^{\prime}$ is the critical argument. Although displayed in Eq. 56 as a function of primed Delaunay variables, it is actually expressed in terms of the variables $A_{j}{ }^{\prime}, E_{j}{ }^{\prime}$, and $I_{j}{ }^{\prime}$, which bear the same functional relationship to the primed Delaunay variables as $A_{j}, E_{j}$, and $I_{j}$ due to the unprimed Delaunay variables through the intermediary of Eqs. 1,33 , and 35 .

The critical argument librates about the value 0 , of course. Letting $\Theta^{\prime}$ be a variable conjugate to $\theta^{\prime}$, the
Hamiltonian takes the form

$$
F^{\prime}=F_{0}{ }^{\prime}+F_{1}{ }^{\prime}\left(\Theta^{\prime}, \theta^{\prime}\right)+F_{2}{ }^{\prime}
$$

where

$$
F_{1}{ }^{\prime}\left(\Theta^{\prime}, \theta^{\prime}\right)=\frac{1}{2} P \Theta^{\prime 2}-Q \cos \theta^{\prime},
$$

$P$ and $Q$ being positive numbers. Here, $F_{0}$ contains only action variables corresponding to the angles that have been eliminated, and $F_{2}$ contains $\theta^{\prime}$ as well as the action variables.

Up to this point all calculations have been in terms of variables which are not canonical. Since the Hori-Lie algorithm (Hori 1966) is canonically invariant, it may be that we could continue to use these variables in the present step; however, we have not seen a simple way to do this and have, therefore, adopted a new canonical set which includes $\left(\Theta^{\prime}, \theta^{\prime}\right)$. We set

$$
\begin{array}{ll}
x_{1}{ }^{\prime}=L_{1}{ }^{\prime}+H_{2}{ }^{\prime} / 2, & x_{4}{ }^{\prime}=L_{2}{ }^{\prime}-H_{2}{ }^{\prime}, \\
x_{2}{ }^{\prime}=G_{1}{ }^{\prime}+H_{2}{ }^{\prime}, & x_{5}{ }^{\prime}=G_{2}{ }^{\prime}-H_{2}{ }^{\prime}, \\
x_{3}{ }^{\prime}=H_{1}{ }^{\prime}+H_{2}{ }^{\prime}, & x_{6}{ }^{\prime}=H_{2}{ }^{\prime} / 2=\Theta^{\prime}+\bar{x}_{6}{ }^{\prime},
\end{array}
$$

where $\bar{x}_{6}{ }^{\prime}$ is a constant defined below, and

$$
\begin{array}{ll}
y_{1}{ }^{\prime}=l_{1}{ }^{\prime}, & y_{4}{ }^{\prime}=l_{2}{ }^{\prime}, \\
y_{2}{ }^{\prime}=g_{1}{ }^{\prime}, & y_{5}{ }^{\prime}=g_{2}{ }^{\prime}, \\
y_{3}{ }^{\prime}=h_{1}{ }^{\prime}, & y_{6}{ }^{\prime}=\theta^{\prime} .
\end{array}
$$

We then introduce new canonical variables $\left(X_{j}{ }^{\prime}, Y_{j}{ }^{\prime}\right)$ by means of the equations

$$
\begin{aligned}
x_{j}{ }^{\prime} & =\bar{x}_{j}{ }^{\prime}+X_{j}{ }^{\prime}, \\
y_{j}{ }^{\prime} & =\quad Y_{j}{ }^{\prime}, \quad j=1, \ldots, 5, \\
\gamma & =(Q / P)^{\frac{1}{2}}, \\
x_{6}{ }^{\prime} & =\bar{x}_{6}{ }^{\prime}+\left(2 X_{6}{ }^{\prime} \gamma\right)^{\frac{1}{2}} \cos Y_{6}{ }^{\prime}, \\
y_{6}{ }^{\prime} & =\quad\left(2 X_{6}{ }^{\prime} / \gamma\right)^{\frac{1}{2}} \sin Y_{6}{ }^{\prime},
\end{aligned}
$$

where $\bar{x}_{j}{ }^{\prime}$ are the nominal values of the $x_{j}{ }^{\prime}$, calculated using the nominal values of the elements introduced previously. $\left(X_{1}{ }^{\prime}, \ldots, X_{5}{ }^{\prime}\right)$ are small deviations analogous to $A_{j}{ }^{\prime}, E_{j}{ }^{\prime}$, and $I_{j}{ }^{\prime}$, and the pair $\left(X_{6}{ }^{\prime}, Y_{6}{ }^{\prime}\right)$ has been chosen in such a way as to be action-angle variables appropriate to the libration of $\left(\Theta^{\prime}, \theta^{\prime}\right)$. The constant $\gamma$ is the nominal value of the mean motion of the libration of the critical argument.

The variables $A_{j}{ }^{\prime}, E_{j}{ }^{\prime}$, and $I_{j}{ }^{\prime}$ are now expressed as series in the variables $\left(X_{1}{ }^{\prime}, \ldots, X_{6}{ }^{\prime}, Y_{6}{ }^{\prime}\right)$, in a manner analogous to Eqs. 23, and are substituted, together with $\theta^{\prime}=y_{6}{ }^{\prime}$, into Eq. 56, expanding in Taylor's series with respect to $\theta^{\prime}$, to obtain a Hamiltonian of the form

$$
\begin{aligned}
\widetilde{F}^{\prime} & =\widetilde{F}_{0}{ }^{\prime}\left(X_{1}{ }^{\prime}, \ldots, X_{5}{ }^{\prime}\right) \\
& +\widetilde{F}_{1}{ }^{\prime}\left(X_{6}{ }^{\prime}\right) \\
& +\widetilde{F}_{2}{ }^{\prime}\left(X_{1}{ }^{\prime}, \ldots, X_{6}{ }^{\prime}, Y_{6}{ }^{\prime}\right),
\end{aligned}
$$

where

$$
\widetilde{F}_{1}^{\prime}=\gamma X_{6}^{\prime}
$$

In practice, the values of $P$ and $Q$, and hence of $\gamma$, are determined as a by-product of this transformation, 
which is carried out for convenience in several steps. However, it could in principle have been carried out in one step as outlined above, by the device of carrying $\gamma$ as an additional literal variable and then adjusting its value to obtain the form of Eq. 63.

In Eq. $63, \widetilde{F}_{2}^{\prime}$ is a periodic function of $Y_{6}{ }^{\prime}$ while $\widetilde{F}_{0}$ is a constant and, therefore, not of importance in the elimination of $Y_{6}{ }^{\prime}$. To eliminate $Y_{6}{ }^{\prime}$ we again make use of the Hori-Lie algorithm and write

$$
\tilde{F}^{\prime \prime}=\frac{1}{2 \pi} \int_{0}^{2 \pi} \tilde{F}^{\prime}\left(X_{j}^{\prime \prime}, Y_{6}^{\prime \prime}\right) d Y_{6}^{\prime \prime},
$$

and

$$
\begin{aligned}
\tilde{F}^{\prime \prime}-\widetilde{F}^{\prime}\left(X_{j}^{\prime \prime}, Y_{6}^{\prime \prime}\right) & =\left\{\widetilde{F}^{\prime \prime}, W^{\prime}\right\} \\
& =\frac{\partial \widetilde{F}^{\prime \prime}}{\partial X_{6}^{\prime \prime}} \frac{\partial W^{\prime}}{\partial Y_{6}^{\prime \prime}},
\end{aligned}
$$

so that the determining function is

$$
W^{\prime}=\left(\frac{\partial \widetilde{F}^{\prime \prime}}{\partial X_{6}^{\prime \prime}}\right)^{-1} \int\left[\tilde{F}^{\prime \prime}-\tilde{F}^{\prime}\left(X_{6}^{\prime \prime}, Y_{6}^{\prime \prime}\right)\right] d Y_{6}^{\prime \prime} .
$$

The series for $\partial \widetilde{F}^{\prime \prime} / \partial X_{6}{ }^{\prime \prime}$ is dominated by the constant $\gamma$ and, therefore, its reciprocal is readily computed by geometric series.

This completes the elimination of the critical argument. The Hamiltonian is now a function of $X_{1}^{\prime \prime}, \ldots, X_{6}{ }^{\prime \prime}$ only, so that these are constants and $Y_{1}^{\prime \prime}, \ldots, Y_{6}^{\prime \prime}$ are linear functions of the time. In the next section we describe the method to be used in computing any desired function of the motion.

\section{CALCULATION OF THE PERTURBATIONS}

Let $p$ be any function of the classical elements of Eqs. 1. The question to be asked is, how can the value of $p$ be computed at any desired time?

Putting aside for the moment the question of the determination of the constants of integration in this theory, let us assume that they are $\left(\bar{X}_{j}^{\prime \prime}, \bar{Y}_{j}^{\prime \prime}\right)$. The evolution of the variables in time is given by

$$
\begin{aligned}
\frac{d X_{j}^{\prime \prime}}{d t} & =\left\{X_{j}^{\prime \prime}, \tilde{F}^{\prime \prime}\right\}=0 \\
\frac{d Y_{j}^{\prime \prime}}{d t} & =\left\{Y_{j}^{\prime \prime}, \tilde{F}^{\prime \prime}\right\} \\
& =-\frac{\partial \widetilde{F}^{\prime \prime}}{\partial X_{j}^{\prime \prime}} \\
& =n_{j}^{\prime \prime}
\end{aligned}
$$

where $n_{j}{ }^{\prime \prime}$ are constants (depending, to be sure, on the constants of integration $\left.\bar{X}_{j}^{\prime \prime}\right)$. Therefore,

$$
\begin{aligned}
& X_{j}{ }^{\prime \prime}=\bar{X}_{j}{ }^{\prime \prime}, \\
& Y_{j}{ }^{\prime \prime}=Y_{j}{ }^{\prime \prime}+n_{j}{ }^{\prime \prime} t
\end{aligned}
$$

gives the time evolution of the doubly primed system. By Hori's algorithm, to first order we have

$$
\begin{gathered}
X_{j}{ }^{\prime}=X_{j}{ }^{\prime \prime}+\left\{X_{j}{ }^{\prime \prime}, W^{\prime}\right\}, \\
Y_{j}{ }^{\prime}=Y_{j}{ }^{\prime \prime}+\left\{{Y_{j}}^{\prime \prime}, W^{\prime}\right\},
\end{gathered}
$$

and since the computation of the Poisson brackets is trivial, these can also be considered to be known as explicit series in $\left(\bar{X}_{j}^{\prime \prime}, \bar{Y}_{j}{ }^{\prime \prime}, t\right)$.

Series expressions for $A_{j}{ }^{\prime}, E_{j}{ }^{\prime}$, and $I_{j}{ }^{\prime}$ have already been derived in terms of $X_{j}{ }^{\prime}, Y_{j}{ }^{\prime}$, so these can be considered to be known. Also, we have from Eqs. 60 and 61

$$
\begin{aligned}
& l_{1}{ }^{\prime}=Y_{1}{ }^{\prime}, \\
& g_{1}{ }^{\prime}=Y_{2}{ }^{\prime}, \\
& h_{1}{ }^{\prime}=Y_{3}{ }^{\prime}, \\
& {l_{2}}^{\prime}=Y_{4}{ }^{\prime}, \\
& g_{2}{ }^{\prime}=Y_{5}{ }^{\prime}, \\
& {h_{2}}^{\prime}=\frac{1}{2} Y_{1}{ }^{\prime}+Y_{2}{ }^{\prime}+Y_{3}{ }^{\prime}-Y_{4}{ }^{\prime}-Y_{5}{ }^{\prime}-\left(X_{6}{ }^{\prime} / 2 \gamma\right)^{\frac{1}{2}} \sin Y_{6}{ }^{\prime},
\end{aligned}
$$

so that $l_{j}{ }^{\prime}, g_{j}{ }^{\prime}$, and $h_{j}{ }^{\prime}$ are known.

Our series manipulation programs are capable of punching out expressions for these formulae, ready to be compiled by a standard FORTRAN compiler. Experience on other contexts (Shelus and Jefferys 1975) has shown that the FORTRAN compiler can be made to produce efficient code for the evaluation of the expressions in question.

With $\left(A_{j}{ }^{\prime}, E_{j}{ }^{\prime}, I_{j}{ }^{\prime}, l_{j}{ }^{\prime}, g_{j}{ }^{\prime}, h_{j}{ }^{\prime}\right)$ in hand, the results of Sec. VIII are now used to compute perturbations in any desired quantity by means of the relation

$$
p\left(A_{j}, \ldots, h_{j}\right)=p\left(A_{j}{ }^{\prime}, \ldots, h_{j}{ }^{\prime}\right)+\left\{p, W_{1}\right\} .
$$

\section{CONSTANTS OF INTEGRATION}

Every theory has its constants of integration, and this one is no exception. The constants $\left(\bar{X}_{j}^{\prime \prime}, \bar{Y}_{j}{ }^{\prime \prime}\right)$ serve in this capacity. As with most theories, the interpretation of the constants is rather obscure, and more so in this case because of the rather complicated sequence of steps required to obtain them.

The determination of the constants to be used in any practical situation is basically inverse to the procedure of Sec. $\mathrm{X}$ of this paper. However, it seems most appropriate to approach it as a problem in differential correction, e.g., assuming approximate values for $\left(\bar{X}_{j}^{\prime \prime}, \bar{Y}_{j}^{\prime \prime}\right)$, we predict values of (say) $r, \psi$, and $\beta$, and compare these with the observed values. By least squares, corrections to the constants are derived, and the process iterated until convergence. This is straightforward, provided that a subroutine for computing, e.g., $r, \psi$, and $\beta$ as functions of any input values of 
$\left(\bar{X}_{j}^{\prime \prime}, \bar{Y}_{j}^{\prime \prime}, M_{j}, \Delta J_{j}, t\right)$ is available. The construction of this subroutine has been discussed above.

Once the constants of integration $\left(\bar{X}_{j}{ }^{\prime \prime}, \bar{Y}_{j}{ }^{\prime \prime}\right)$ have been determined, it is straightforward to relate them to such quantities as the osculating values of the elements. Indeed, one of the elements (say $a_{j}$ ) can be considered just another function $p$ of the variables $\left(A_{j}, \ldots, h_{j}\right)$; applying Eq. 71 , it can be expressed in terms of the constants of integration. In this way, the present theory can (in principle, at least) be compared with any other theory.

\section{PRESENT AND FUTURE STATUS OF THE THEORY}

At present, we have computed explicit expressions for a preliminary theory through Sec. $\mathrm{X}$ of this paper. The preliminary theory has been computed with more truncation than we expect to use in the definitive theory, and is currently being tested against numerical integrations. At the same time, a differential correction procedure is being programmed, as outlined in Sec. XI.

When they become available, the series of new observations (Abbot, Mulholland, and Shelus 1975) are to be combined with the old ones, and the definitive constants of the theory determined. As stated before, the theory (available in its final form as a set of
FORTRAN subroutines) is to be literal, in that it is valid for a range of values of the important parameters and constants of integration.

\section{ACKNOWLEDGMENTS}

The support of the National Aeronautics and Space Administration under grant NGR 44-012-282, is gratefully acknowledged. The participation of Dr. J. Derral Mulholland and Dr. Peter Shelus in numerous helpful conversations has been an important contribution to this work.

\section{REFERENCES}

Abbot, R., Mulholland, J. D., and Shelus, P. J. (1975). To be published.

Barton, D. (1966). Astron. J. 71, 438.

Broucke, R. (1971). Celest. Mech. 4, 497.

Hori, G.-I. (1966). Publ. Astron. Soc. Japan 18, 287.

Jefferys, W. H. (1970). Celest. Mech. 2, 467.

Jefferys, W. H. (1972). Celest. Mech. 6, 117.

Jefferys, W. H., and Moser, J. (1966). Astron. J. 71, 568.

Lieske, J. H. (1974). Astron. Astrophys. 31, 137.

Mulhòlland, J. D. (1972). Remarks made at the Outer Planet Workshop, Jet Propulsion Laboratory, Pasadena, 11-12 October.

Shelus, P. J., and Jefferys, W. H. (1975). Celest. Mech. 11, 75. Struve, G. (1930). Veröff. UUniv. Berlin-Babelsberg 6, No. 4, 61. Struve, G. (1933). Veröff. Univ. Berlin-Babelsberg 6, No. 5, 10. Woltjer, J. (1928). Ann. Sterrewacht Leiden 16, Pt. 3, 64. 